\section{Excluding myeloma diagnosis using revised thresh- olds for serum free light chain ratios and M-protein levels}

In Western Europe in 2016 there were 35,000 new cases of and 22,000 deaths from multiple myeloma. ${ }^{1}$ Myeloma is one of the worst cancers for delays in diagnosis and half of patients saw their primary physician three or more times before referral to a hematologist. ${ }^{2}$ Population data from England show that a third of 22,249 newly diagnosed myeloma patients presented as emergencies and had the worst 12-month survival at just $61 \% .^{3}$ Myeloma is suspected when patients present with one or more features of myeloma-related end-organ damage including anemia, skeletal disease, renal impairment and immunodeficiency but any of these are more likely caused by much commoner diseases. Myeloma is rare in primary care consultations and the symptoms are nonspecific, common and of low predictive value (odds ratios $\leq 3)$. ${ }^{4,5}$

Critical to the diagnosis of myeloma is testing serum for the presence of an $\mathrm{M}$-protein (paraprotein) plus serum and/or urine testing for monoclonal free light chains (FLC). Absence of an M-protein and abnormal serum FLC ratio is only found in the rare non-secretory myeloma, and therefore with close to $100 \%$ sensitivity for diagnosis of myeloma, encouraging widespread use of these simple blood tests may reduce delays in myeloma diagnosis. ${ }^{6}$ However, ordering these tests is tempered because they also reveal the 100 times more common condition monoclonal gammopathy of undetermined significance (MGUS). In clinical practice the majority of M-proteins and abnormal serum FLC ratios derive from MGUS plasma cell clones or are small abnormalities in the ratio caused by conditions unrelated to neoplastic plasma cells, including kidney disease, inflammation and infection. Myeloma arises in an age range in which these conditions are common and there is a need to define serum FLC ratio reference ranges better in these groups of patients. ${ }^{8-10}$ MGUS is not usually clinically significant and not the cause of the patients presenting illness but requires differentiation from myeloma and this can be difficult. ${ }^{11}$ Particularly in primary care and other specialties there may be unnecessary rapid referrals to hematologists, inappropriate testing (imaging or bone marrow biopsies), and associated anxiety in patients. This is a significant problem in the UK where patients are frequently referred unnecessarily as urgent suspected cases of cancer and contributes to gross inefficiency within healthcare systems that are struggling with capacity. There is a lack of guidance for non-hematologists in interpretation of abnormal test results in this setting. It would be useful to have evidence-based guidelines on laboratory reporting of M-protein levels and serum FLC ratios that would enable primary care and other specialty doctors to decide which patients to refer urgently to hematology for suspected myeloma.

We examined the use of different $M$-protein level thresholds combined with different serum FLC ratio ranges to distinguish myeloma from MGUS without loss of sensitivity for identifying patients who need urgent referral for myeloma diagnosis and assessment. Central laboratory serum FLC ratios and M-protein concentrations in 3,177 newly diagnosed myeloma patients from UK clinical trials ${ }^{12,13}$ and 711 MGUS cases were analyzed. ${ }^{7}$

The distribution of $\mathrm{M}$-protein levels for myeloma patients with an IgG or IgA M-protein (accounting for $84 \%$ of all myeloma patients) are shown in Figure 1 . IgG patients had significantly higher M-protein levels (median $37 \mathrm{~g} / \mathrm{L}$, range $0.80-129 \mathrm{~g} / \mathrm{L}$ ) compared to IgA patients (median $34.5 \mathrm{~g} / \mathrm{L}$, range $0.2-109 \mathrm{~g} / \mathrm{L})(P<0.001)$ likely reflecting the longer serum half-life of IgG. A cutoff of 30 $\mathrm{g} / \mathrm{L}$ is used to separate MGUS from smoldering (asymptomatic) myeloma. In this large cohort of newly diagnosed myeloma patients who required anti-myeloma therapy $33 \%$ of $\operatorname{IgG}$ patients and $43 \%$ of $\operatorname{IgA}$ patients had $\mathrm{M}$-proteins $<30 \mathrm{~g} / \mathrm{L}$. Five percent of IgG patients and $11 \%$ of IgA patients presented with an $\mathrm{M}$-protein level $<10 \mathrm{~g} / \mathrm{L}$, which is below the threshold recommended for accurate assessment of $M$-protein response to therapy.

Patients were classified as having a normal or abnormal

Table 1. Table 1. The percentage of myeloma patients who would be undetected at diagnosis according to various serum free light chain $\kappa: \lambda$ ratio ranges and M-protein level thresholds.

\begin{tabular}{|c|c|c|c|c|c|c|}
\hline \multirow[b]{2}{*}{$\kappa: \lambda$ ratio ranges } & \multicolumn{3}{|c|}{$\begin{array}{l}\text { Percentage and number of patients missed } \\
\text { if using FLC ratio alone to detect myeloma }\end{array}$} & \multicolumn{3}{|c|}{$\begin{array}{l}\text { Percentage and number of patients missed using } \\
\text { both FLC ratio and M-protein level }\end{array}$} \\
\hline & $\begin{array}{l}\text { All patients } \\
(\mathrm{N}=3177)\end{array}$ & $\begin{array}{l}\text { Light chain only } \\
(n=436,13.8 \%)\end{array}$ & $\begin{array}{c}\lg G / A / M / D \\
(n=2717,86.2 \%)\end{array}$ & $\begin{array}{c}\text { M-protein } \\
<5 \mathrm{~g} / \mathrm{L} \\
(\mathrm{n}=122)\end{array}$ & $\begin{array}{l}\text { M-protein } \\
<10 \mathrm{~g} / \mathrm{L} \\
(\mathrm{n}=226)\end{array}$ & $\begin{array}{c}\text { M-protein } \\
<15 \mathrm{~g} / \mathrm{L} \\
(\mathrm{n}=336)\end{array}$ \\
\hline Normal ratio range & $5.2 \%$ & 0 & $4.7 \%$ & $0.4 \%$ & $0.7 \%$ & $0.9 \%$ \\
\hline$(0.26-1.65)$ & (172) & & (148) & (12) & (23) & (29) \\
\hline Extended ratio range 1 & $10 \%$ & $0.06 \%$ & $9.3 \%$ & $0.5 \%$ & $1.1 \%$ & $1.6 \%$ \\
\hline$(0.15-3.36)$ & (319) & (2) & (293) & (16) & (35) & (49) \\
\hline Extended ratio range 2 & $15 \%$ & $0.16 \%$ & $14.2 \%$ & $0.5 \%$ & $1.3 \%$ & $2.1 \%$ \\
\hline$(0.08-7.41)$ & (478) & $(5)$ & $(449)$ & (16) & $(40)$ & $(65)$ \\
\hline Proposed reference range & $13.8 \%$ & $0.13 \%$ & $13.0 \%$ & $0.5 \%$ & $1.2 \%$ & $2.0 \%$ \\
\hline$(0.1-7.0)$ & $(438)$ & $(4)$ & $(410)$ & (16) & (38) & $(62)$ \\
\hline
\end{tabular}

Patients were classified as having a normal serum free light chain (FLC) ratio according to the following reference ranges: (i) normal range for serum FLC ratio (0.26-1.65); (ii) an extended ratio range that encompassed $10 \%$ of all myeloma patients at disease presentation (0.15-3.36); (iii) an extended ratio range that encompassed $15 \%$ of all myeloma patients at disease presentation (0.08-7.41) and (iv) a proposed reference range simplified for ease of use in clinical practice (0.1-7.0) For each of these serum FLC ratio ranges, patients with a normal ratio were identified and then categorized according to myeloma type and, in the three columns on the right, according to M-protein level. Of 436 patients with only light chains, 18 had oligosecretory myeloma (immunofixation-negative in serum and urine but abnormal serum FLC ratio and FLC levels sufficient to measure response to therapy) and 24 patients ( $0.8 \%$ of the total 3,177 patients) were non-secretors defined by immunofixation-negative blood and urine and serum FLC ratio within the normal reference range; these were excluded from the analysis. The percentages are those of all patients with secretory disease ( $\mathrm{n}=3,153$ ). 


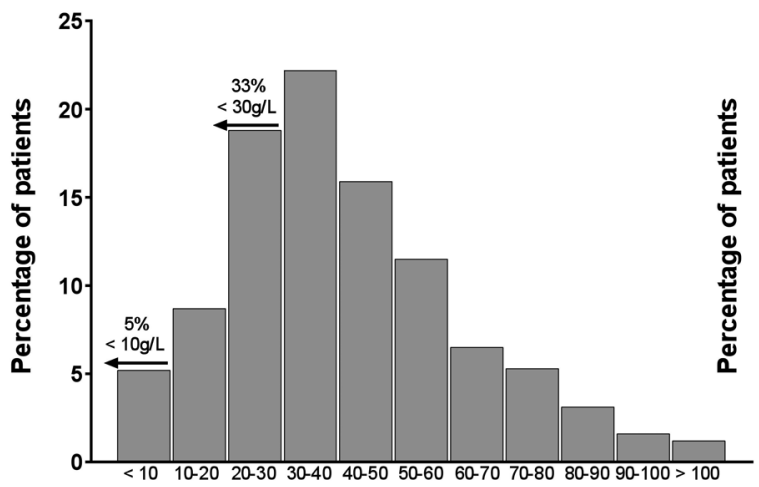

$\operatorname{lgG}$ M-protein (g/L)

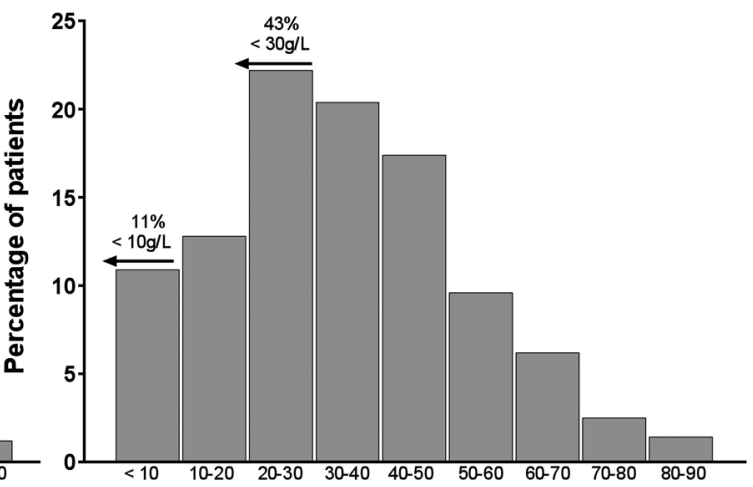

$\lg A$ M-protein (g/L)

Figure 1. Distribution of M-protein levels in IgG and IgA myeloma patients at entry into the Myeloma IX and Myeloma XI clinical trials. Data are shown for patients with IgG ( $n=1,894$, left panel) and IgA ( $n=757$, right panel) multiple myeloma at first diagnosis. A sizable proportion of these patients with symptomatic multiple myeloma presented with M-protein levels $<30 \mathrm{~g} / \mathrm{L}$, used as part of the criteria to separate patients with monoclonal gammopathy of undetermined significance $(<30 \mathrm{~g} / \mathrm{L})$ and asymptomatic (smoldering) myeloma ( $>30 \mathrm{~g} / \mathrm{L})$. Furthermore, a significant proportion of those patients presented with M-protein levels of $<10 \mathrm{~g} / \mathrm{L}$; the threshold for accurate assessment of response to therapy. M-protein levels were significantly higher in patients with IgG M-proteins than in those with IgA M-proteins $(P<0.001$, Mann-Whitney U-test).

serum FLC ratio according to the diagnostic ratio reference range (NRR) of $0.26-1.65,{ }^{14}$ as per the International Myeloma Working Group (IMWG) guidelines for the diagnosis of all plasma cell dyscrasias. ${ }^{15}$ To investigate alternative reference ranges, all trial patients' serum FLC ratios at myeloma diagnosis were rank ordered. Five percent of myeloma patients had a serum FLC ratio within the NRR. Serum FLC ratio ranges were then extended outwards to encompass $10 \%$ then $15 \%$ of all patients at diagnosis. Two-thirds of myeloma patients have $\kappa$ FLC and one third $\lambda$ FLC. To reflect this, the extended $\kappa \lambda$ reference ranges were placed above 1.65 to add two-thirds of the additional patients as $\kappa$ and below 0.26 to add onethird of additional patients as $\lambda$. This generated an extended reference range 1 of $0.15-3.36$ and an extended reference range 2 (ERR2) of 0.08-7.41.

Twenty-four patients ( $0.8 \%$ of the total 3,177 patients) were non-secretors defined by immunofixation negative blood and urine and a serum FLC ratio within the NRR; these patients were excluded from the analysis. Patients with secretory disease (M-protein and/or FLC, $n=3,153$ ) were stratified by a normal serum FLC ratio according to each of the three reference ranges (NRR 0.26-1.65; ERR1 0.15-3.36; ERR2 0.08-7.41). When these reference ranges were used alone, the serum FLC ratio failed to identify $5.2 \%, 10 \%$ and $15 \%$ of all newly diagnosed myeloma patients, respectively (Table 1). For the 436 patients who had an abnormal serum FLC ratio and no $M$-protein [light chain only $(n=418)$ and oligosecretory $(\mathrm{n}=18)$ patients], ERR1 missed two patients and ERR2 missed five patients. For the 2,717 patients with an IgG/A/M or D M-protein, the serum FLC ratio test alone missed $4.7 \%, 9.2 \%$ and $14.4 \%$ for the NRR, ERR1 and ERR2, respectively.

Patients secreting an $\mathrm{M}$-protein were then subdivided into those with or without an $\mathrm{M}$-protein level of $<5 \mathrm{~g} / \mathrm{L}$, $<10 \mathrm{~g} / \mathrm{L}$ and $<15 \mathrm{~g} / \mathrm{L}$ (Table 1). The use of the three serum FLC ratio ranges combined with these specified $M$-protein levels greatly enhanced sensitivity for the identification of new myeloma. Using the NRR, just $0.4-0.9 \%$ of all patients were missed when using both biomarkers across the different $M$-protein cut-points. In the same way the broader ERR1 missed $0.5-1.6 \%$ and the broadest ERR2 missed $0.5-2.1 \%$ of all new myeloma patients. For ease of use in clinical practice, a simplified ratio may be most appropriate. We propose $0.1-7.0$, which, as shown in Table 1, yielded very similar percentages and numbers to EER2. These data demonstrate that, combined with an $\mathrm{M}$-protein threshold of $10 \mathrm{~g} / \mathrm{L}$, significantly widening the serum FLC ratio reference range from the NRR of 0.26 1.65 to $0.1-7.0$ only decreased sensitivity for identification of new myeloma by $0.5 \%$.

We next applied these extended serum FLC ratio ranges and $M$-protein thresholds to a cohort of 711 MGUS patients (484 IgG, 109 IgA and 118 IgM), to assess how this strategy could be used to exclude MGUS patients from urgent in-depth myeloma investigations. As shown in Table 2, use of the serum FLC ratio on its own excluded $53.9 \%$ (NRR) up to $88.9 \%$ (EER2) of MGUS patients. Combining serum FLC ratios with the three $\mathrm{M}$-protein cut-points $(<5,<10,<15 \mathrm{~g} / \mathrm{L}), 68.8 \%$, to $90.1 \%$ MGUS patients were excluded using the NRR and $89.5 \%$ to $95.5 \%$ were excluded using the simplified serum FLC ratio range of $0.1-7.0$

The use of the serum FLC ratio range of $0.1-7.0$ in combination with an $\mathrm{M}$-protein threshold of $10 \mathrm{~g} / \mathrm{L}$ included $97.9 \%$ of myeloma cases and excluded $93.4 \%$ of MGUS cases. The $2.1 \%$ of myeloma patients missed $(n=66)$ included $0.8 \%$ of non-secretors. Using other commercially available assays, such as $\mathrm{N}$ Latex (Siemens, Germany) and Seralite (Abingdon Health, UK) these greatly widened serum FLC ratio ranges are thus likely to provide similar sensitivity and specificity for distinguishing myeloma from MGUS but this needs to be tested.

A similar process combining the use of $\mathrm{M}$-protein levels plus serum FLC ratio has provided a useful tool for risk stratification of MGUS patients for progression to myeloma. ${ }^{7}$ Our proposed model adds an additional instrument to aid clinicians in differentiating patients at high risk that myeloma is the cause of their presenting illness from those more likely to have another disease causing their presenting illness with coincidental MGUS. This risk stratification should not, of course, be used in isolation and should be applied in conjunction with clinical symptoms and other laboratory biomarkers. The use of this instrument may enable the wider application of myeloma screening in patients presenting with features of myeloma-related end-organ damage. This may be 
Table 2. The percentage of 711 patients with monoclonal gammopathy of undetermined significance that would be excluded from further assessment for myeloma using the stated cutoffs for reference ranges for serum free light chain $\kappa: \lambda$ ratio and ratio ranges combined with M-protein level thresholds

\begin{tabular}{lcccc}
\multicolumn{2}{c}{$\begin{array}{c}\text { Percentage and number of patients excluded } \\
\text { if using FLC ratio alone } \\
\text { All patients } \\
(\mathbf{n = 7 1 1 )}\end{array}$} & $\begin{array}{c}\text { Percentage and number of patients excluded if applying sFLC } \\
\text { ratio and M-protein level } \\
\text { M-protein }\end{array}$ & $\begin{array}{c}\text { M-protein } \\
<\mathbf{1 5} \mathrm{g} / \mathrm{L}\end{array}$ \\
Normal ratio range & $53.9 \%$ & $68.8 \%$ & $81.2 \%$ & $90.1 \%$ \\
$(0.26-1.65)$ & $(383)$ & $(468)$ & $(577)$ & $(646)$ \\
Extended ratio range 1 & $77.8 \%$ & $81.7 \%$ & $88.6 \%$ & $93.2 \%$ \\
$(0.15-3.36)$ & $(553)$ & $(581)$ & $(630)$ & $(663)$ \\
\hline Extended ratio range 2 & $88.9 \%$ & $90.7 \%$ & $94.2 \%$ & $96.2 \%$ \\
$(0.08-7.41)$ & $(632)$ & $(645)$ & $(670)$ & $(684)$ \\
Proposed reference range & $87.2 \%$ & $89.5 \%$ & $93.4 \%$ & $95.5 \%$ \\
$(0.1-7.0)$ & $(620)$ & $(636)$ & $(664)$ & $(679)$
\end{tabular}

Patients were classified as having a normal serum free light chain $(\mathrm{FLC})$ ratio according to the four different reference ranges and the percentage of patients that would be excluded from further assessment for myeloma is shown for each serum FLC ratio range. The columns on the right incorportate those with a normal ratio, then those with an abnormal ratio who can then be further excluded based on the three M-protein levels

important to attenuate the delays in myeloma diagnosis which negatively affects patients' outcomes. In conclusion, applying an M-protein threshold of $10 \mathrm{~g} / \mathrm{L}$ combined with a serum FLC ratio range of 0.1-7 excludes $93.4 \%$ of MGUS cases and provides $97.9 \%$ sensitivity for the detection of myeloma. This sensitivity is just $0.5 \%$ less than that achieved using the five-fold narrower range of $0.26-1.65$ that has a poor specificity for myeloma.

Jennifer L. J. Heaney, ${ }^{1}$ Alex Richter, ${ }^{1}$ Stella Bowcock, ${ }^{2}$ Guy Pratt, ${ }^{3}$ J. Anthony Child, Graham Jackson, ${ }^{4}$ Gareth Morgan, ${ }^{6}$ Ingemar Turesson ${ }^{7}$ and Mark T. Drayson ${ }^{1}$

'Institute of Immunology and Immunotherapy, University of Birmingham, Birmingham, UK; ${ }^{2}$ King's College Hospital NHS Trust, London, UK; ${ }^{3}$ University Hospitals Birmingham NHS Trust, Birmingham, UK; ${ }^{4}$ University of Leeds, Leeds, UK; ${ }^{5}$ University of Newcastle, Newcastle, UK; ${ }^{6}$ Perlmutter Cancer Center, New York, NY, USA and 'Lund University, Lund, Sweden

Acknowledgments: we are grateful to the National Cancer Research Institute Hematooncology subgroup and to all principal investigators for their dedication and commitment to recruiting patients to the Myeloma IX and Myeloma XI trials. We thank the Clinical Trials Research Unit at The University of Leeds (Leeds, UK; Myeloma IX and XI). We are grateful to the staff of the Clinical Immunology Service in Birmingham and Tim Plant, Karen Walker, Alison Adkins, and Nicola Newnham. We are also grateful to all patients and their clinical teams at centres throughout the UK whose participation made this study possible. The Department of Clinical Chemistry at Skane University Hospital provided stored serum samples for the Swedish MGUS study and we thank the investigators involved in the original data collection of the MGUS study.

Correspondence: MARK T. DRAYSON

m.t.drayson@bham.ac.uk

doi:10.3324/haematol.2019.224360

Information on authorship, contributions, and financial \& other disclosures was provided by the authors and is available with the online version of this article at WWw. haematologica.org.

\section{References}

1. Cowan AJ, Allen C, Barac A, et al. Global burden of multiple myeloma: a systematic analysis for the global burden of disease study 2016. JAMA Oncol. 2018;4(9):1221-1227.
2. Neal RD, Din NU, Hamilton W, et al. Comparison of cancer diagnostic intervals before and after implementation of NICE guidelines: analysis of data from the UK general practice Research Database. Br J Cancer. 2014;110(3):584-592.

3. National Cancer Registration and Analysis Service Public Health England. Routes to diagnosis 2006-2015 Workbook Version 2.0b; Available from: www.ncin.org.uk/view? rid=3549

4. Shephard EA, Neal RD, Rose P, Walter FM, Litt EJ, Hamilton WT. Quantifying the risk of multiple myeloma from symptoms reported in primary care patients: a large case-control study using electronic records. Br J Gen Pract. 2015;65(631)e106-113.

5. Goldschmidt N, Zamir L, Poperno A, Kahan NR, Paltiel O. Presenting signs of multiple myeloma and the effect of diagnostic delay on the prognosis. J Am Board Fam Med. 2016;29(6):702-709.

6. Heaney JLJ, Campbell JP, Griffin AE, et al. Diagnosis and monitoring for light chain only and oligosecretory myeloma using serum free light chain tests. Br J Haematol. 2017;178(2):220-230.

7. Turesson I, Kovalchik SA, Pfeiffer RM, et al. Monoclonal gammopathy of undetermined significance and risk of lymphoid and myeloid malignancies: 728 cases followed up to 30 years in Sweden. Blood. 2014;123(3):338-345.

8. Hutchison CA, Plant T, Drayson M, et al. Serum free light chain measurement aids the diagnosis of myeloma in patients with severe renal failure. BMC Nephrol. 2008;9(11):1471-2369.

9. Campbell JP, Heaney JL, Shemar M, et al. Development of a rapid and quantitative lateral flow assay for the simultaneous measurement of serum kappa and lambda immunoglobulin free light chains (FLC): inception of a new near-patient FLC screening tool. Clin Chem Lab Med. 2017;55(3):424-434.

10. Zemlin AE, Ipp H, Rensburg MA, et al. Serum free light chains in patients with HIV infection: their association with markers of disease severity and antiretroviral use. J Clin Pathol. 2015;68(2):148153.

11. Rajkumar SV, Lacy MQ, Kyle RA. Monoclonal gammopathy of undetermined significance and smoldering multiple myeloma. Blood Rev. 2007;21(5):255-265

12. Morgan GJ, Davies FE, Gregory WM, et al. First-line treatment with zoledronic acid as compared with clodronic acid in multiple myeloma (MRC Myeloma IX): a randomised controlled trial. Lancet. 2010;376(9757):1989-1999.

13. Jackson GH, Davies FE, Pawlyn C, et al. Lenalidomide maintenance versus observation for patients with newly diagnosed multiple myeloma (Myeloma XI): a multicentre, open-label, randomised, phase 3 trial. Lancet Oncol. 2019;20(1):57-73.

14. Katzmann JA, Clark RJ, Abraham RS, et al. Serum reference intervals and diagnostic ranges for free kappa and free lambda immunoglobulin light chains: relative sensitivity for detection of monoclonal light chains. Clin Chem. 2002;48(9):1437-1444.

15. Dispenzieri A, Kyle R, Merlini G, et al. International Myeloma Working Group guidelines for serum-free light chain analysis in multiple myeloma and related disorders. Leukemia. 2009;23(2): 215-224. 